\title{
Gamma Reaction History for the NIF*
}

\author{
H. W. Herrmann, S. H. Batha, S. C. Evans, Y. Kim, \\ J. M. Mack, C. S. Young \\ Los Alamos National Laboratory \\ Los Alamos, 87455 NM, USA
}

B. C. Cox, B. C. Frogget, M. I. Kaufman, R. M. Malone,

T. W. Tunnell

National Security Technologies,

Los Alamos, NM 87544, USA

\author{
W. Stoeffl \\ Lawrence Livermore National Laboratory, \\ Livermore, CA 94551, USA \\ C. J. Horsfield \\ Atomic Weapons Establishment \\ Aldermaston, Reading, RG7 4PR, UK
}

Bang time and reaction history measurements are fundamental components of diagnosing ICF implosions and will be essential contributors to diagnosing attempts at ignition on the National Ignition Facility (NIF). Fusion gammas provide a direct measure of fusion interaction rate without being compromised by Doppler spreading. Gamma-based gas Cherenkov detectors that convert fusion gamma rays to optical Cherenkov photons for collection by fast recording systems have been developed and fielded at Omega. These systems have established their usefulness in illuminating ICF physics in several experimental campaigns. Bang time precision better than 25 ps has been demonstrated, well below the 50 ps accuracy requirement defined by the NIF System Design Requirements. A staged approach of implementing Gamma Reaction History (GRH) diagnostics on the NIF has been initiated. In the early stage, multiple detectors located close to target chamber center (at 2 and $6 \mathrm{~m}$ ) and coupled to photomultiplier tubes are geared toward the loweryield THD campaign. In the later stage, streak cameracoupled instruments will be used for improved temporal resolution at the higher yields expected from the DT ignition campaign. Multiple detectors will allow for increased dynamic range and gamma energy spectral information.

\footnotetext{
* Work supported by US DOE/NNSA, performed by LANL, operated by LANS LLC under Contract DE-AC5206NA25396.
} 